\title{
Expression levels of serum miRNA-195 in different types of patients with cholangiocarcinoma and its value to determine the prognosis thereof
}

\author{
QINGLIANG CHEN ${ }^{1}$, CONGMEI WANG $^{1}$, HONGKAI ZHANG $^{2}$, YUN LI $^{3}$, YUNHONG CAO $^{1}$, \\ YONGXIANG ZHANG ${ }^{1}$, SHAOKUN LIU ${ }^{1}$, ZHEN LI $^{4}$, XIAOGUO XIN ${ }^{1}$ and XINWEI HAN ${ }^{4}$ \\ ${ }^{1}$ Department of Radiology, The Third People's Provincial Hospital of Henan Province, Zhengzhou, Henan 450006; \\ ${ }^{2}$ Department of Radiology, Henan Cancer Hospital, Zhengzhou, Henan 450008; ${ }^{3}$ Department of Radiology, \\ Zhengzhou No. 7 People's Hospital, Zhengzhou, Henan 450016; ${ }^{4}$ Department of Radio-Intervention, \\ The First Affiliated Hospital of Zhengzhou University, Zhengzhou, Henan 450052, P.R. China
}

Received August 18, 2017; Accepted January 8, 2018

DOI: $10.3892 / \mathrm{ol} .2018 .8016$

\begin{abstract}
This study aimed to investigate the expression levels of microRNA-195 (miRNA-195) in different types of patients with cholangiocarcinoma (CCA) and its correlation with the prognosis. Serum samples were collected from different types of patients with CCA (I, II, III, IV) and normal cases, followed by detection of expression of miRNA-195 using quantitative polymerase chain reaction (qPCR). The serum samples of 204 patients with CCA, including 75 cases of type I, 68 cases of type II, 35 cases of type III and 26 cases of type IV and 200 healthy subjects were selected. The baseline clinicopathological data of patients with CCA were assessed and recorded, and patients were followed up constantly. The receiver operating characteristic (ROC) curve was established, and the area under the ROC curve (AUC) was calculated to evaluate the difference of miRNA-195 expression levels between patients with CCA and normal controls. Survival curves were set up for groups with high and low expression levels via the Kaplan-Meier method, and the log-rank test was used to evaluate the difference of survival curves between the two groups. The expression of miR-195 in patients with CCA was significantly lower than that in the normal control group, with a sensitivity of 0.78 and a specificity of 0.76 , and it was positively correlated with the pathological grade of CCA. Additionally, the expression level of serum miRNA-195 was associated with lymph node metastasis $(\mathrm{P}=0.009)$ and tumor-node-metastasis (TNM) classification $(\mathrm{P}=0.010)$. The survival analysis revealed that the prognosis in patients with CCA in types III and IV was poorer than that in those with types I and III who had a
\end{abstract}

Correspondence to: Professor Xinwei Han, Department of RadioIntervention, The First Affiliated Hospital of Zhengzhou University, 1 Jianshe Dong Road, Zhengzhou, Henan 450052, P.R. China E-mail: hanxinwei2017@163.com

Key words: miRNA, cholangiocarcinoma, prognosis, $\mathrm{qPCR}, \mathrm{ROC}$ low expression of miRNA-195 ( $\mathrm{P}=0.0026)$. The results show that miR-195 is an important marker that reflects the malignant degree of CCA, and it is expected to be a reference marker to determine the prognosis of CCA.

\section{Introduction}

Cholangiocarcinoma (CCA) is a highly aggressive tumor derived from bile duct epithelial cells. In recent decades, the incidence and mortality rate of CCA have been on the increase worldwide $(1,2)$. The survival rate and prognosis of CCA patients are not ideal due to the early invasion and metastasis (3). Although radical surgery is the only therapy for CCA, the disease is difficult to be controlled and treated given that patients are diagnosed in the middle or late stage when the disease is identified (4). Thus, it is imperative to further understand the pathogenesis of CCA, in order to search for a new diagnostic and therapeutic method $(5,6)$.

MicroRNA (miRNA) is a type of endogenous, small, non-coding single-stranded RNA molecule that regulates the expression of target genes after its transcription, and it is involved in the regulation of multiple physiological and pathological functions (7). Previous findings have shown that the abnormal expression of miRNA-195 is associated with breast cancer (8), lung cancer (9) and many other tumors, but the role of miRNA-195 in CCA has not been reported yet. Therefore, this study aimed to investigate the expression level and prognostic value of miRNA-195 in different pathological grades of CCA, and to provide a direction for further exploration of the potential targets of CCA gene therapy.

\section{Materials and methods}

Clinical data. In total, 204 CCA patients admitted to the Third People's Provincial Hospital of Henan Province (Zhengzhou, China) from March, 2009 to May, 2015 were selected. CCA patients with different pathological types were divided into two groups using magnetic resonance imaging (MRI), according to the Bismuth-Corlette typing method. In groups of type I and II, 
Table I. Reverse transcription of miRNA-195 and RT-PCR primers.

Item

Primer sequence

miRNA-195

Reverse transcription primer

Upstream primers

Downstream primers

GAPDH

Upstream primers

Downstream primers

\section{5'-CTCAACTGGTGTCGTGGAGTCGGCAATTCAGTTGAGGCCAATAT-3' \\ 5'-ACACTCCAGCTGGGTA GCAGCACAGAAAT-3' \\ 5'-TGGTGTCGTGGAGTCG-3'}

there were 59 males and 74 females, aged 56-68 years, with an average age of $62.2 \pm 65.7$ years. In groups of type III and IV, there were 30 males and 41 females, aged 65-73 years, with an average age of $69.3 \pm 73.8$ years. In addition, 200 healthy subjects examined at the Third People's Provincial Hospital of Henan Province were enrolled as the control group. There were no statistically significant differences in age and sex in each group $(\mathrm{P}<0.05)$.

The study was approved by the Ethics Committee of the Third People's Provincial Hospital of Henan Province, and informed consent was obtained from the selected subjects or family members. The study was in line with the Medical Ethics Standards.

Collection of samples. Peripheral venous blood $(2.5 \mathrm{ml})$ was collected from the elbow of patients, followed by anticoagulation using ethylene diaminetetraacetic acid dipotassium (EDTA-K2) and preserved in a refrigerator at $-80^{\circ} \mathrm{C}$. EDTA-K2 anticoagulant blood $(2 \mathrm{ml})$ was extracted by the transfer liquid gun and added into a centrifuge tube, followed by centrifugation at $1,260 \mathrm{x}$ g at room temperature for $10 \mathrm{~min}$. Then, the serum was taken and placed into the high-speed centrifuge for centrifugation at $8,000 \mathrm{x}$ g for $10 \mathrm{~min}$. Finally, the serum was collected and reserved in a refrigerator at $-80^{\circ} \mathrm{C}$.

Main reagents. The experimental material TRIzol reagent was purchased from Thermo Fisher Scientific (Waltham, MA, USA); Deoxyribonuclease I (DNase I) (RNase-free) reagent was purchased from Thermo Fisher Scientific; and ReverTra Ace qPCR RT kit and SYBR-Green Real-Time PCR Master Mix were purchased from Thermo Fisher Scientific. The internal primers were produced by Shanghai Invitrogen Biotechnology Co., Ltd., Shanghai, China (Table I).

Reverse transcription of $m R N A$ into complementary DNA (cDNA). miRNA of total RNA was reversely transcribed into cDNA according to the protocol of the miRNA reverse transcription kit. The reaction conditions of reverse transcription of miRNA-195 were: $16^{\circ} \mathrm{C}$ for $30 \mathrm{~min}, 42^{\circ} \mathrm{C}$ for $30 \mathrm{~min}$ and $85^{\circ} \mathrm{C}$ for $5 \mathrm{~min}$; the reaction conditions and system of internal reference glyceraldehyde-3-phosphate dehydrogenase (GAPDH) were conducted according to the instructions of ReverTra Ace qPCR RT kit. Subsequently, three duplicate wells were made in all the samples.
Data analysis. The fluorescence signals of each reaction well were collected quantitatively, and the $\mathrm{Cq}$ values were recorded. The expression of miRNA-195 in CCA tissue relative to the normal tissue adjacent to the tumor was expressed as $2^{-\Delta \Delta \mathrm{Cq}} . \Delta \mathrm{Cq}$ is the difference of $\mathrm{Cq}$ values of the target gene to the internal reference GAPDH in each sample, namely $\Delta \mathrm{Cq}=\mathrm{Cq}\left({ }_{\text {miR-195 }}\right)-\mathrm{Cq}\left({ }_{\text {GAPDH }}\right), \Delta \Delta \mathrm{Cq}=\left(\mathrm{Cq}_{\text {miR-195 }}-\mathrm{Cq}_{\mathrm{GAPDH}}\right)$ tumor $-\left(\mathrm{Cq}_{\text {miR-195 }}-\mathrm{Cq}_{\mathrm{GAPDH}}\right)_{\text {normal }} . \Delta \Delta \mathrm{Cq}<1$ indicated that the expression level of miR-195 in the bile duct tissue was lower than that in the paired pericancerous tissues, while $\Delta \Delta \mathrm{Cq}>1$ indicated that the expression level of miR-195 in bile duct tissue was higher than that in paired pericancerous tissues.

Statistical analysis. Statistical Product and Service Solutions 21.0 statistical software (SPSS, Inc., Chicago, IL, USA) was used to process data in this experiment. Enumeration data were analyzed using the Chi-square test. Measurement data with normal distribution were expressed as mean \pm SD and analyzed by Student's t-test. Pearson's correlation analysis was used for the determination of the correlation of the expression of miR-195 with clinical features of CCA. P $<0.05$ was considered to indicate a statistically significant difference.

\section{Results}

Expression levels of miR-195 in the serum of CCA patients and healthy controls. In this experiment, the results of the detection of miR-195 expression levels in the serum of CCA patients and healthy controls, qPCR showed that the expression level of miR-195 in the serum of CCA patients $(1.671 \pm 0.955)$ was lower than that of in the serum of healthy controls (3.574 \pm 1.751$)$, and the difference was statistically significant ( $\mathrm{t}=3.237, \mathrm{P}=0.014$ ) (data not shown).

Expression levels of miR-195 among the different groups of patients with CCA. In this experiment, CCA patients with different pathological degrees were divided into two groups (I, II; III, IV). The expression level was $2.874 \pm 0.612$ in Group I and II and 1.985 \pm 0.434 in Group III and IV (data not shown). The results indicated that the expression level was distinctly reduced in the groups with high pathological degree (III, IV) compared with that in the groups with low pathological degree (I, II). In addition, the receiver operating characteristic (ROC) curve analysis revealed that the value of miR-195 in predicting the risk of CCA was high, the area 
Table II. Clinicopathological features of patients with CCA.

\begin{tabular}{lrc}
\hline Clinicopathological features & Case & Relative expression level of miR-195 (I, II; III, IV) \\
\hline Age (years) & 120 & $2.443 \pm 0.874 ; 2.034 \pm 0.385$ \\
$<60$ & 84 & $2.351 \pm 0.752 ; 1.985 \pm 0.543$ \\
$\geq 60$ & & \\
Sex & 9 & $2.364 \pm 0.494 ; 1.821 \pm 0.610$ \\
Male & 115 & $2.211 \pm 0.529 ; 1.901 \pm 0.577$ \\
Female & 155 & \\
Degree of differentiation & 49 & $2.089 \pm 0.564 ; 1.964 \pm 0.341$ \\
Well & & $1.936 \pm 0.436 ; 1.794 \pm 0.386$ \\
Moderate + Poor & 140 & $1.842 \pm 0.428 ; 1.754 \pm 0.367$ \\
TNM staging & 64 & $1.695 \pm 0.614 ; 1.681 \pm 0.315$ \\
I/II & & \\
III/IV & 34 & $1.684 \pm 0.447 ; 1.594 \pm 0.541$ \\
Lymph node metastasis & 170 & $1.867 \pm 0.761 ; 1.710 \pm 0.611$ \\
Positive & & 0.068 \\
Negative & &
\end{tabular}

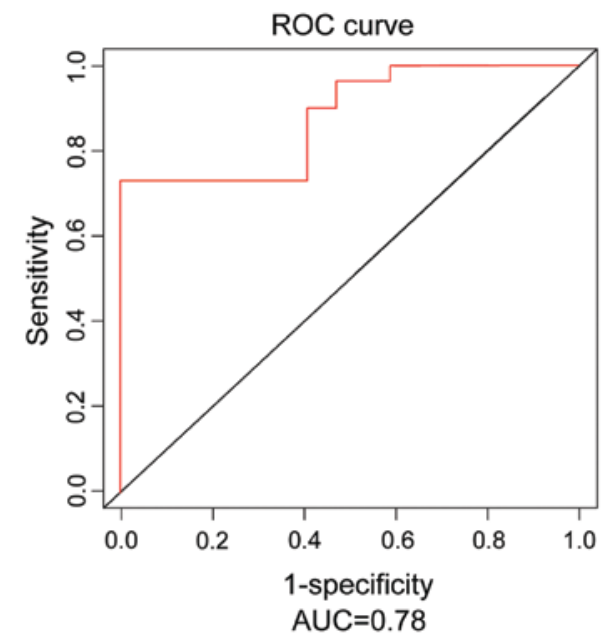

Figure 1. ROC curves of miR-195 in the serum of CCA and the normal control group. The value of miR-195 in predicting the risk of CCA is high, AUC is 0.78 , and $95 \%$ confidence interval (CI) is $0.729-0.834$.

under the ROC curve (AUC) was 0.78 , and $95 \%$ confidence interval (CI) was 0.729-0.834 (Fig. 1).

Relationship of the expression level of miRNA-195 in serum with the clinicopathological features of patients with CCA. The relationship of the expression of miRNA-195 with the clinicopathological features [sex, age, degree of differentiation and tumor-node-metastasis (TNM) staging] of patients with CCA were analyzed. According to the design in this study, the CCA patients were divided into two groups (I, II; III, IV), and the results indicated that the expression level of the serum miRNA-195 was closely associated with TNM staging and lymph node metastasis. The expression of miRNA-195 in the serum was gradually decreased with the increase of pathological staging, and the differences had statistical significance

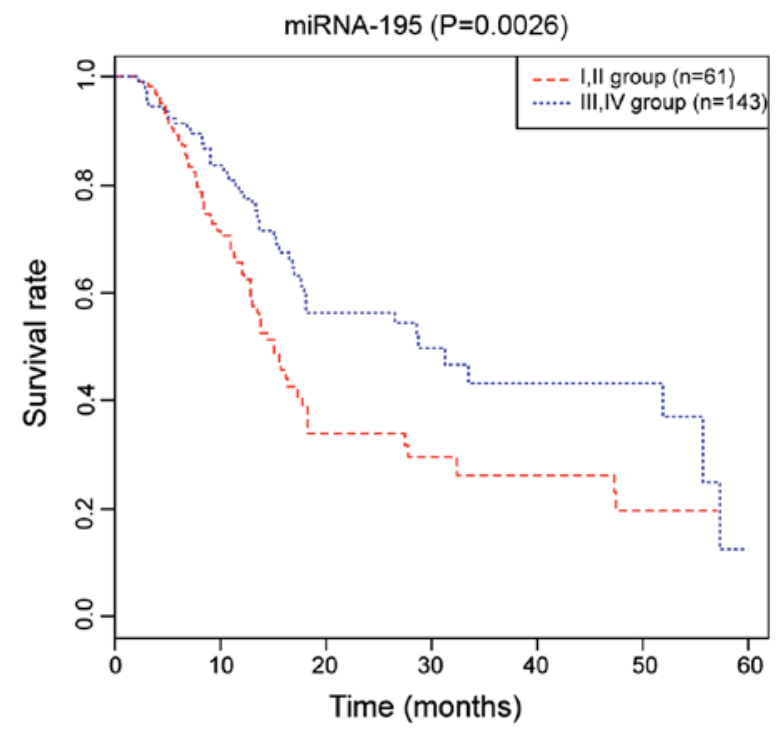

Figure 2. The Kaplan-Meier survival curves of the miRNA-195 Group I and II (61 patients) as well as Group III and IV (143 patients) among CCA patients. The 5-year survival rate in Group I and II is higher than that in Group III and IV among CCA patients $(\mathrm{P}=0.0026)(\mathrm{P}<0.05)$.

$\left(\chi^{2}=6.547, \mathrm{P}=0.010 ; \chi^{2}=7.147, \mathrm{P}=0.009\right)$. There were no correlations between the expression of miRNA-195 and the serum and clinical features of CCA including age, sex and degree of differentiation, and the differences were not statistically significant (Table II).

Relationship of miRNA-195 with the 5-year survival rate of the patients with CCA. The results of the Kaplan-Meier survival curve analysis indicated that 5-year survival rate in the serum miRNA-195 Group I and II was higher than that in Group III and IV, and the difference was statistically significant $(\mathrm{P}=0.0026)$ (Fig. 2). 


\section{Discussion}

At present, MRI and computed tomography (CT) are common imaging methods used in the diagnosis of CCA, but the disease cannot be diagnosed in the early stage, thus leading to missed diagnosis and misdiagnosis easily. Therefore, this study aimed to diagnose and prevent disease through miRNAwhich is regarded as an independent prognostic factor. Numerous studies have shown that miRNA is abnormally expressed in some tumors, such as breast cancer (10), rectal cancer (11), lung cancer (12) and thyroid carcinoma (13) and can be used as an effective diagnostic index and assures stable detection. In addition, miRNA is a tumor marker that has broad prospects and can indicate the tumor status to some extent $(14,15)$.

By detecting and comparing expression levels of miRNA-195 between the CCA patients and healthy controls in this study, it was found that miRNA-195 was downregulated in the CCA patients compared with that in the healthy controls. The study demonstrated that miRNA-195 is a diagnostic index that has certain sensitivity and specificity in CCA patients. In this study, the expression of miRNA-195 in the serum and clinical features of CCA patients was analyzed, resulting in the serum miRNA-195 being closely associated with TNM staging and lymph node metastasis, which may be due to its inhibitory effect on the migration and metastasis of tumor cells. In addition, there was no correlation with age, sex and degree of differentiation. The relationship of the expression of miRNA-195 in the serum with the 5-year survival rate in patients with CCA was analyzed in this study. The results showed that the serum of miRNA-195 Group I and II was higher than that in Group III and IV.

miRNA-195 is a non-coding RNA molecule with a length of 22 nucleotides, which is closely related to the occurrence and progression of various tumors. miRNA-195 has the tissue and space specificity, which can promote or inhibit cancer genes in different types of cancers, thus playing different roles. Previous findings have shown that miR-195 inhibited cancer metastasis in vitro and in vivo by partially targeting cell cycle gene CyclinD1 (CCND1) in osteosarcoma thus inhibiting the occurrence of cancer (16). Additionally, miR-195 was downregulated in hepatocellular carcinoma and adrenocortical carcinoma as a tumor suppressor (17). However, it was upregulated in chronic lymphocytic leukemia and breast cancer as an oncogene $(10,18,19)$.

The present study investigated the expression of miRNA-195 in the serum of CCA patients and its clinical value in the diagnosis and prognosis of CCA, suggesting that serum miRNA-195 was highly expressed in CCA, which had a certain specificity. Notably, the expression of miRNA-195 in the serum was positively associated with the clinical analysis in patients with CCA, which had a relatively high clinical value in diagnosis and prognosis. This study further demonstrated that the expression of miRNA was differentially expressed, and it is required to explore its specific molecular mechanism in exerting the role as an oncogene in CCA.

In summary, the results of the present study have shown that miRNA-195 is lowly expressed in the serum of CCA patients compared with that in the control group. miRNA-195 in serum can be used as an effective diagnostic index for CCA. The expression of miRNA-195 in serum is closely related to the clinical staging of the CCA patients and the 5-year survival rate in serum miRNA-195 Group I and II is higher than that in Group III and IV. The present study lays a certain foundation for further research on the mechanism of occurrence and progression of CCA, and provides a potential target for the early diagnosis and prognosis of CCA.

\section{Acknowledgements}

Not applicable.

\section{Funding}

This study was supported by Henan Medical Science and Technology Project. Project name: Measurement and clinical application of 3.0T high field 3D MRCP biliary anatomy. Project no. 201503218. Project manager Qingliang Chen.

\section{Availability of data and materials}

The datasets used and/or analyzed during the current study are available from the corresponding author on reasonable request.

\section{Authors' contributions}

QC and CW analyzed and interpreted the patient data, and were the major contributors in writing the manuscript. $\mathrm{HZ}$ and ZL performed the experiment and participated in the design of the study. YL participated in the analysis and discussion of the data. YC and XX were responsible for the collection of the data and the follow-up management of the patients. YZ was responsible for the statistical analysis of the data. SL and $\mathrm{XH}$ were the major contributors in designing the methods. All authors read and approved the final manuscript.

\section{Ethics approval and consent to participate}

This study was approved by the Ethics Committee of the Third People's Provincial Hospital of Henan Province. Signed written informed consents were obtained from the patients and/or guardians. The study was in line with the Medical Ethics Standards.

\section{Consent for publication}

Not applicable.

\section{Competing interests}

The authors declare that they have no competing interests.

\section{References}

1. Blechacz B: Cholangiocarcinoma: Current knowledge and new developments. Gut Liver 11: 13-26, 2017.

2. Marcano-Bonilla L, Mohamed EA, Mounajjed T and Roberts LR: Biliary tract cancers: Epidemiology, molecular pathogenesis and genetic risk associations. Linchuang Zhongliuxue Zazhi 5: 61, 2016.

3. Razumilava N and Gores GJ: Cholangiocarcinoma. Lancet 383: 2168-2179, 2014.

4. Fairweather M, Balachandran VP and D'Angelica MI: Surgical management of biliary tract cancers. Linchuang Zhongliuxue Zazhi 5: 63, 2016. 
5. Høgdall D, O'Rourke CJ, Taranta A, Oliveira DV and Andersen JB: Molecular pathogenesis and current therapy in intrahepatic cholangiocarcinoma. Dig Dis 34: 440-451, 2016.

6. Jiang XM, Li ZL, Li JL, Zheng WY, Li XH, Cui YF and Sun DJ: LncRNA CCAT1 as the unfavorable prognostic biomarker for cholangiocarcinoma. Eur Rev Med Pharmacol Sci 21: 1242-1247, 2017.

7. Zen K and Zhang CY: Circulating microRNAs: A novel class of biomarkers to diagnose and monitor human cancers. Med Res Rev 32: 326-348, 2012.

8. Luo Q, Wei C, Li X, Li J, Chen L, Huang Y, Song H, Li D and Fang L: MicroRNA-195-5p is a potential diagnostic and therapeutic target for breast cancer. Oncol Rep 31: 1096-1102, 2014.

9. Yongchun Z, Linwei T, Xicai W, Lianhua Y, Guangqiang Z, Ming Y, Guanjian L, Yujie L and Yunchao H: MicroRNA-195 inhibits non-small cell lung cancer cell proliferation, migration and invasion by targeting MYB. Cancer Lett 347: 65-74, 2014.

10. Heneghan HM, Miller N, Kelly R, Newell J and Kerin MJ: Systemic miRNA-195 differentiates breast cancer from other malignancies and is a potential biomarker for detecting noninvasive and early stage disease. Oncologist 15: 673-682, 2010.

11. Eriksen AHM, Sørensen FB, Andersen RF, Jakobsen A and Hansen TF: Association between the expression of microRNAs and the response of patients with locally advanced rectal cancer to preoperative chemoradiotherapy. Oncol Lett 14: 201-209, 2017.

12. Takamizawa J, Konishi H, Yanagisawa K, Tomida S, Osada H, Endoh H, Harano T, Yatabe Y, Nagino M, Nimura Y, et al: Reduced expression of the let-7 microRNAs in human lung cancers in association with shortened postoperative survival. Cancer Res 64: 3753-3756, 2004.

13. Forte S, La Rosa C, Pecce V, Rosignolo F and Memeo L: The role of microRNAs in thyroid carcinomas. Anticancer Res 35: 2037-2047, 2015.
14. Letelier P, Riquelme I, Hernández AH, Guzmán N, Farías JG and Roa JC: Circulating MicroRNAs as biomarkers in biliary tract cancers. Int J Mol Sci 17: 17, 2016.

15. Ortiz-Quintero B: Cell-free microRNAs in blood and other body fluids, as cancer biomarkers. Cell Prolif 49: 281-303, 2016.

16. Han K, Chen X, Bian N, Ma B, Yang T, Cai C, Fan Q, Zhou Y and Zhao TB: MicroRNA profiling identifies MiR-195 suppresses osteosarcoma cell metastasis by targeting CCND1. Oncotarget 6: 8875-8889, 2015.

17. Soon PS, Tacon LJ, Gill AJ, Bambach CP, Sywak MS, Campbell PR, Yeh MW, Wong SG, Clifton-Bligh RJ, Robinson BG, et al: MiR-195 and miR-483-5p identified as predictors of poor prognosis in adrenocortical cancer. Clin Cancer Res 15: 7684-7692, 2009.

18. Zanette DL, Rivadavia F, Molfetta GA, Barbuzano FG, ProtoSiqueira R, Silva-Jr WA, Falcão RP and Zago MA: miRNA expression profiles in chronic lymphocytic and acute lymphocytic leukemia. Braz J Med Biol Res 40: 1435-1440, 2007.

19. Hannafon BN, Sebastiani P, de las Morenas A, Lu J and Rosenberg CL: Expression of microRNA and their gene targets are dysregulated in preinvasive breast cancer. Breast Cancer Res 13: R24, 2011.

(i) (9) This work is licensed under a Creative Commons Attribution-NonCommercial-NoDerivatives 4.0 International (CC BY-NC-ND 4.0) License. 